\title{
Kann Levetiracetam die Überlebenszeit verlängern?
}

Fragestellung: Die Autoren untersuchten, ob der Einsatz von Levetiracetam als Antikonvulsivum bei Patienten mit neu diagnostiziertem Glioblastom die Überlebenszeit verlängert.

Hintergrund: Operation gefolgt von konkomitanter und Erhaltungschemotherapie mit Temozolomid zusätzlich zur Strahlentherapie ist die Standardbehandlung bei neu diagnostiziertem Glioblastom. Seit Jahren halten sich hartnäckig Hypothesen, dass die Wahl des Antikonvulsivums bei Patienten mit symptomatischer Epilepsie das Überleben beeinflusst. Speziell für eine Assoziation einer Valproinsäuretherapie mit besserer Prognose liegen zahlreiche Publikationen vor.

Kim YH, Kim T, Joo JD et al. Survival benefit of levetiracetam in patients treated with concomitant chemoradiotherapy and adjuvant chemotherapy with temozolomide for glioblastoma multiforme. Cancer 2015; May 14. doi: $10.1002 /$ cncr.29439
Patienten und Methodik: Die Autoren untersuchten die Krankengeschichten von 103 Patienten, von denen 58 (56\%) Levetiracetam für mindestens drei Monate erhalten hatten. Mittels Cox
Regression wurde eine multivariate Analyse durchgeführt, die Alter, Resektionsausmaß, Karnofsky Performance Score (KPS) und O6-Methylguanin-DNA-Methyltransferase (MGMT)-Promoter-Methylierungsstatus einschloss.

Ergebnisse: Das mediane progressionsfreie Überleben und das Gesamtüberleben für die mit Levetiracetam behandelten Patienten betrug 9,4 Monate beziehungsweise 25,7 Monate. Es war damit signifikant länger als für Patienten, die kein Levetiracetam erhielten (progressionsfreies Überleben 6,7 Monate, Gesamtüberleben 16,7 Monate). In der multivariaten Analyse blieben präoperativer KPS, MGMT-Promotor-Methylierungsstatus und Levetiracetamtherapie signifikant mit der Prognose assoziiert.

Schlussfolgerungen: Die Autoren mutmaßen, dass die Zunahme von Levetiracetam die Überlebenszeit beim Glioblastom verlängern könnte und verweisen als mögliche Erklärung unter anderem auf eine sensibilisierende Wirkung für die TemozolomidTherapie, die nach einer präklinischen Untersuchung über eine MGMT-Hemmung zustande kommen soll.

\section{- Kommentar von Michael Weller, Zürich, Schweiz}

\section{Bis zum Vorliegen eindeutiger Daten auf Primärprophylaxe verzichten}

Nachdem bisher vor allen Dingen die zusätzliche Einnahme von Valproinsäure mit einem besseren Überleben beim Glioblastom in Verbindung gebracht wurde, liegen jetzt ähnliche Daten für Levetiracetam vor. Wie auch die Daten für Valproinsäure sollten die Daten für Levetiracetam nicht überbewertet werden, sie rechtfertigen keinesfalls den Einsatz von Levetiracetam ohne entsprechende Indikation aufgrund einer symptomatischen Epilepsie. Durch die Forderung, dass diese Patienten für mindestens drei Monate Levetiracetam einnahmen, wird ein Bias in die Analyse eingeführt, weil solche Patienten, zumindest über einen gewissen Zeitraum, offenkundig einen stabilen Krankheitsverlauf hatten. Des Weiteren bleibt offen, inwieweit eine symptomatische Epilepsie zur Indikationsstellung für Antikonvulsiva führte. Die symptomatische Epilepsie

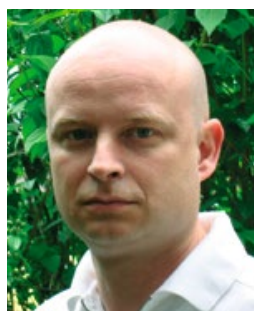

Prof. Dr. med. Michael Weller,

Zürich/Schweiz

Direktor der Klinik für Neurologie,

Universitätsspital Zürich

E-Mail: michael.weller@usz.ch per se wurde zumindest in einigen Studien mit einem längeren Überleben in Verbindung gebracht, möglicherweise im Sinne eines Lead-in-Bias aufgrund früherer Diagnose. Eine differenzierte Analyse der Bedeutung von symptomatischer Epilepsie versus Therapie mit Levetiracetam war offensichtlich nicht möglich und wäre auch methodisch angesichts einer retrospektiven Analyse relativ weniger Patienten anspruchsvoll. In dieser Situation sollte bis zum Vorliegen prospektiv erhobener, gut kontrollierter Daten zur Beeinflussung des Krankheitsverlaufs durch Antikonvulsiva weiterhin bei der Empfehlung geblieben werden, auf eine Primärprophylaxe zu verzichten und die Indikation zu einer einmal eingeleiteten Therapie im Krankheitsverlauf immer wieder infrage zu stellen.

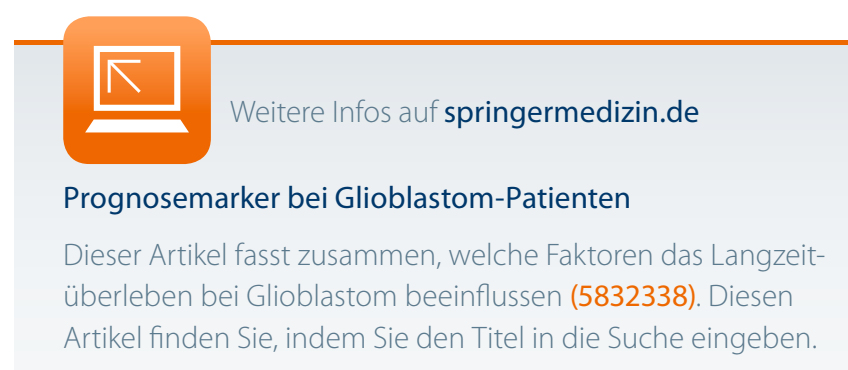

